\title{
Erratum to: Biological evaluation of the toxicity and the cell cycle interruption by some benzimidazole derivatives
}

\author{
Katarzyna Błaszczak-Świątkiewicz ${ }^{1}$. Joanna Sikora ${ }^{2}$ - Jacek Szymański ${ }^{3}$. \\ Marian Danilewicz $^{4} \cdot$ Elżbieta Mikiciuk-Olasik $^{5}$
}

Published online: 4 April 2016

(C) International Society of Oncology and BioMarkers (ISOBM) 2016

Erratum to: Tumor Biol.

DOI 10.1007/s13277-016-4828-1

The original version of this article contained a mistake.

The part B of Figs. 2 and 3 were published erroneously.

The corrected Figs. 2 and 3 are shown in the next page.

The online version of the original article can be found at http://dx.doi.org/ 10.1007/s13277-016-4828-1.

Katarzyna Błaszczak-Świątkiewicz

katarzyna.blaszczak-swiatkiewicz@umed.lodz.pl

1 Department of Pharmacy, Medical University, Muszynskiego 1, 90-151 Lodz, Poland

2 Laboratory of Bioanalysis, Department of Pharmaceutical Chemistry, Drug Analysis and Radiopharmacy, Medical University, Muszynskiego 1, 90-151 Lodz, Poland

3 Central Scientific Laboratory, Medical University, Mazowiecka 6/8, 92-215 Lodz, Poland

4 Educational Center of the Medical University of Lodz, Pomorska 251, 92-213 Lodz, Poland

5 Department of Pharmaceutical Chemistry, Drug Analysis and Radiopharmacy, Medical University, Muszynskiego 1,

90-151 Lodz, Poland 
a

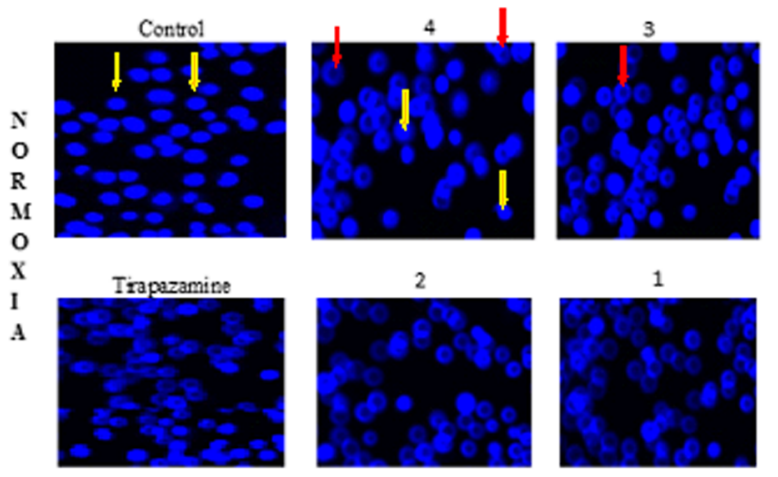

b

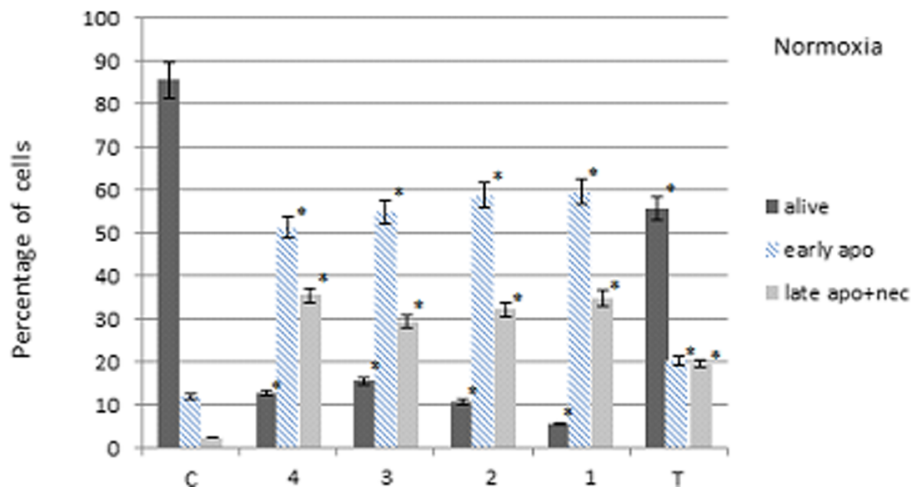

${ }^{*} p<0.05$ (mean $\left.+\mathrm{SD} ; n=3\right)$, yellow arrows indicate normal cells, and red arrows indicate apoptosis cells ( $c$ control, $T$ tirapazamine, compounds 14, apo apoptosis, nec necrosis)
Fig. 2 Visualization of apoptotic cells treated with the tested derivatives and tirapazamine in normoxia. The percentage of different cell populations identified by Hoechst (part A) and PI/Annexin V (part B) assay. a

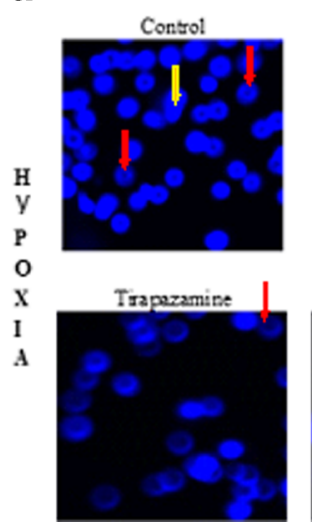

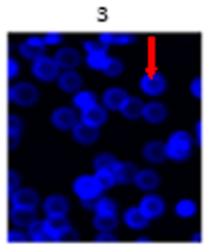
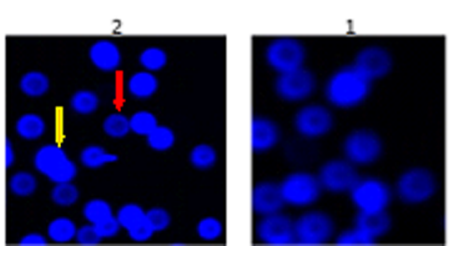

Fig. 3 Visualization of apoptotic cells treated with the tested derivatives and tirapazamine in hypoxia. The percentage of different cell populations identified by Hoechst (part A) and PI/Annexin V (part B) assay. ${ }^{*} p<0.05$

b

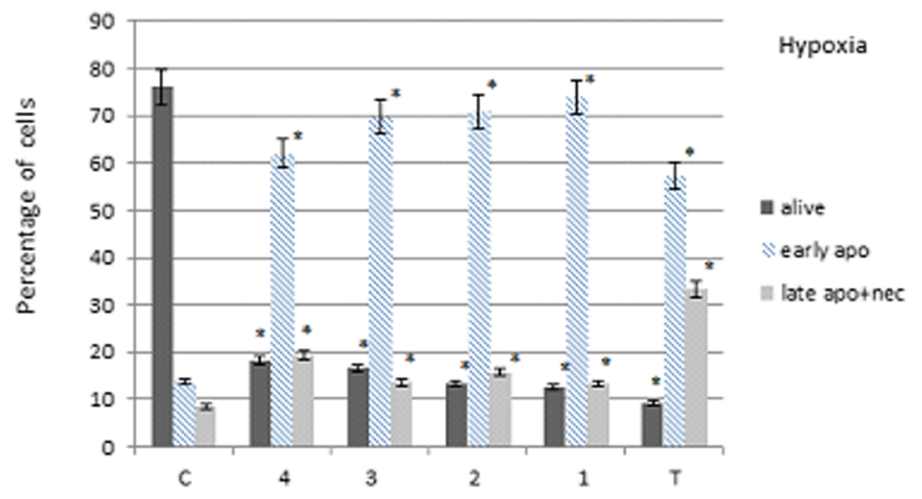

(mean $+\mathrm{SD}$ for three independent experiments; $n=3)$, yellow arrows indicate normal cells, and red arrows indicate apoptosis cells ( $c$ control, $T$ tirapazamine, compounds $\mathbf{1 - 4}$, apo apoptosis, nec necrosis) 